\title{
Influencia del género y la edad en la formación continua multidisciplinar de los docentes de cooperativas de enseñanza
}

\section{Influence of gender and age in the multidisciplinary continuous training of teachers of teaching cooperatives}

Jesús López Belmonte

Antonio José Moreno Guerrero

Santiago Pozo Sánchez

Universidad de Granada, España

Autor para correspondencia: jesuslopezbelmonte@gmail.com, ajmoreno@ugr.es, santiagopozosanchez@gmail.com

Fecha de recepción: 20 de julio de 2018 - Fecha de aceptación: 30 de agosto de 2018

Resumen: Los docentes que desarrollan su profesión en cooperativas de enseñanza deben llevar a cabo un proceso de formación complementaria a los estudios iniciales universitarios y de carácter multidisciplinar para conseguir alcanzar el factor de calidad deseado por estas entidades educativas. El objeto de este estudio se centra en conocer cómo influye el género y la edad del profesorado que conforman las cooperativas de enseñanza en la formación continua. Para efectuar esta investigación se ha seguido un método cuantitativo, a través de un análisis descriptivo y correlativo. Tomando una muestra de 60 docentes pertenecientes a cooperativas de enseñanza de la Ciudad Autónoma de Ceuta. Esta investigación ha determinado que estos docentes otorgan relevancia a la formación continua, manifestando no tener tiempo para ello. Consideran no tener una formación de ámbito empresarial necesaria para gestionar este tipo de instituciones educativas y además no es el tipo de formación que reclaman, sino que buscan formarse en ámbitos pedagógicos. Este colectivo se decanta por una formación presencial, más destacada en los sujetos de más de 50 años, siendo el género femenino quien realiza más tareas formativas. Se concluye que el género y la edad no influyen en la acción formativa de estos profesionales.

Palabras Claves: formación continua; docentes, cooperativas de enseñanza; calidad Abstract: Teachers who develop their profession in educational cooperatives must carry out a training process complementary to initial university studies and multidisciplinary in order to achieve the quality factor desired by these educational entities. The purpose of this study is to know how the gender and age of the teachers that make up the teaching cooperatives in continuing education influence. To carry out this research, a quantitative method has been followed, through a descriptive and correlative analysis. Taking a sample of 60 teachers belonging to teaching cooperatives of the Autonomous City of Ceuta. This research has determined that these teachers give relevance to ongoing training, stating that they do not have time to do so. They consider that they do not have the necessary entrepreneurial training to manage this type of educational institutions, and it is not the kind of training that they claim, but that they seek to train in 
pedagogical fields. This group opts for face-to-face training, more prominent in subjects over 50 years, with the female gender who performs more training tasks. It is concluded that gender and age do not influence the formative action of these professionals.

Key Words: continuous training; teachers; teaching cooperatives; quality

\section{Introducción}

En un entorno que evoluciona rápidamente como consecuencia de los cambios originados por la tecnología dentro de nuestra sociedad, la formación inicial que reciben en las universidades los profesionales de la educación no resulta suficiente para alcanzar una enseñanza eficaz y de calidad, atendiendo a las particularidades que presentan los discentes en la actualidad. Esta situación ha desembocado en la necesidad de una continua actualización del cuerpo docente para ejercer su vocación y sus funciones plenamente (Marcelo, 2002). Toda actividad que suponga un ejercicio de renovación, adquisición y ampliación de conocimientos, habilidades y competencias de los docentes va a englobar al concepto de formación que se está tratando en este trabajo (Nieto y Alfageme, 2017).

En los últimos años se ha generado una elevada preocupación e interés por la formación del profesorado, convirtiéndose en objeto de estudio de investigaciones en el campo de la educación, con la finalidad de mejorar la calidad de la enseñanza, situando al docente como factor elemental de los procesos didácticos (Mayorga, Santos y Madrid, 2014; González, Gento y Orden, 2016).

Hoy en día, la formación permanente e integral del profesorado es entendida como un elemento de gran importancia, puesto que en sus destrezas, sabiduría y conocimientos está el futuro de la sociedad, al tener que transmitir toda su ciencia a las nuevas generaciones (Noguera, 2017).

Siguiendo a González y Barba (2014), hay que concebir a la formación continua como un bucle de aprendizaje permanente durante el desarrollo profesional y laboral de los docentes. Este tipo de formación debe sustentarse en la mejora del perfil de los educadores que contribuya a reducir las prácticas tradicionales y el acomodamiento que se produce en esta profesión. González y Cutanda (2017) añaden que la formación de naturaleza continuada debe configurarse de tal modo que los docentes adquieran el aprendizaje necesario para propiciar una evolución de su praxis diaria.

El reciclaje y renovación de la formación académica de los docentes debe estar orientada hacia la búsqueda de la indagación, el espíritu crítico y la autonomía del profesorado para no caer en las redes de la monotonía y rutina diaria, ocasionando un aumento de la desmotivación y frustración laboral (Andreucci, 2012).

Expertos como San Martín, Peirats y Waliño (2017) consideran que muchos docentes siguen aplicando una metodología tradicional y desactualizada, no aprovechando las potencialidades de los recursos tecnológicos que se dispone hoy día. Para progresar profesionalmente es necesario un cambio de actitud y concienciarse de que la formación complementaria forma parte de la vida de los docentes. 
Analizando los estudios de Lobato y Madinabeitia (2011); Pontes, Serrano y Poyato (2013); Alfaro, Fernández y Alvarado (2014); Gisbert y Lázaro (2015), los temas que más han sido solicitados en los centros de formación del profesorado han sido los referentes a aspectos psicopedagógicos, innovación, nuevas tecnologías, herramientas para la creación de recursos interactivos, nuevas metodologías, técnicas de estudio, idiomas, atención a la diversidad, resolución de conflictos en el aula, orientación académica del alumnado, aspectos formales de la programación docente, relaciones socio-afectivas en la comunidad educativa, primeros auxilios en el entorno escolar y fomento de hábitos saludables en los discentes, entre otros.

Para llevar a cabo una formación continua y constante en el tiempo es fundamental la motivación del profesorado. Los motivos que destacan los autores citados anteriormente junto con Muñoz-Repiso y Murillo (2010) se centran en la promoción profesional, ampliación del campo de conocimientos, adaptación de la enseñanza a la realidad actual, establecimiento de redes y contacto con otros docentes, intercambio de experiencias, búsqueda de calidad en la enseñanza, inversión del tiempo libre y ampliación del currículum vitae.

En este sentido adquiere gran repercusión y relevancia el fenómeno del liderazgo pedagógico, como vía para potenciar y promocionar las capacidades individuales de cada uno de los componentes del claustro. La figura del líder pedagógico se centra en la preocupación acerca de la formación complementaria de los miembros de su alrededor, fomentando e incentivando que tales profesionales realicen tareas formativas que contribuyan al crecimiento y desarrollo profesional, consiguiendo una escuela más efectiva y dinámica (González, Gento y Orden, 2016).

La educación actual demanda y necesita un incremento de la calidad ofertada. Este concepto abstracto e intangible solo se consigue mediante al desarrollo profesional de los docentes por medio de procesos de formación complementaria a sus estudios iniciales en la universidad. Además, se insta a que el profesorado adquiera competencias reflexivas sobre su práctica docente, analizando en consecuencia los aspectos a mejorar para conseguir satisfacer las necesidades que la sociedad demanda (Casanova, 2012). La formación continua de los profesionales que se dedican a la educación ha resultado ser un factor relevante para la obtención de la calidad y adecuación a las singularidades de una sociedad en constante proceso de cambio. Esta formación de naturaleza complementaria supone un refuerzo de la identidad docente y dota de sentido sus quehaceres cotidianos para la consecución de una institución escolar eficaz (Nieto y Alfageme, 2017). Otros autores como Escudero, Cutanda y Trillo (2017) también afirman que el aprendizaje permanente de los docentes y su desarrollo profesional son aspectos importantes y destacados para lograr la calidad en los procesos de enseñanza-aprendizaje.

Según Montes y Torres (2015), aquellas personas dedicadas a la enseñanza que siguen un proceso de formación continua en su trayectoria profesional son concebidas como profesionales comprometidos e implicados en su organización educativa. Para San Martín, Peirats y Waliño (2017), el profesorado, para crecer profesionalmente, debe desarrollar a través de la formación continua un sentido creativo, original e innovador en las acciones desplegadas en la escuela.

Todo docente debe disponer de ciertas habilidades y destrezas para lograr un desempeño de su profesión de manera satisfactoria. Se hace alusión a la capacidad de transmisión de 
conocimientos, motivar el aprendizaje de los discentes, despertar el sentimiento de cooperación y trabajo en equipo, originar una actitud crítica sobre su labor diaria y asumir el cambio metodológico que está experimentando la enseñanza a través de la innovación educativa (Martínez, 2004).

El docente de hoy se encuentra ante una serie de desafíos como la participación activa del alumno, siendo el principal protagonista y eje por el que circula la enseñanza, la inclusión de las TIC en la escuela y la atención a la diversidad que ha originado la convergencia de distintas culturas en la sociedad. Para ello resulta imprescindible y necesaria la formación continua del profesorado en distintos ámbitos de actuación que demanda la educación de la sociedad del conocimiento (García, 2015).

Cada vez son más las tareas y gestiones que se realizan en los centros educativos por medio de la tecnología, es por ello que estos profesionales deben estar formados para la utilización eficiente de todos los recursos que nos brinda la inclusión tecnológica (San Martín, Peirats y Waliño, 2017).

Concretamente, los profesionales de la educación que desempeñan su vocación docente en cooperativas de enseñanza se encuentran ante el reto o la dificultad de gestionar y liderar un centro educativo desde dos perspectivas diferentes, la educativa y la empresarial, puesto que son entidades dentro del campo de la educación, pero pertenecientes a la economía social. Es por ello que el profesorado que conforma esta tipología de centros deben adquirir y desarrollar una formación continua tanto en el plano de la enseñanza como en el ámbito de la gestión y administración empresarial. Además, las cooperativas de enseñanza, entre sus fines y principios, persiguen un modelo de escuela basado en la calidad, innovación y eficacia; y esto solo es posible alcanzarlo siguiendo un plan de formación permanente del claustro de profesores (Fuentes, 2004; López, 2017; López y Fuentes, 2018).

\section{Objetivo de la investigación}

Los objetivos de la presente investigación son:

- Identificar la influencia del género en la formación continua en los docentes de las cooperativas de enseñanza.

- Identificar la influencia de la edad de los docentes de cooperativas de enseñanza en la formación continua.

\section{Método}

\section{Sujetos}

El estudio presentado da continuidad a una investigación realizada en las Cooperativas de Enseñanza de la Ciudad Autónoma de Ceuta. Los centros educativos participantes han sido tres centros concertados (Beatriz de Silva, San Daniel y Severo Ochoa), en los que se imparten las etapas de educación infantil, educación primaria y educación secundaria obligatoria. 
En la investigación se ha utilizado toda la población $(n=60)$, en la que prevalece el género femenino (75\%) sobre el masculino (25\%), cuyas edades se sitúan principalmente por encima de los 50 años de edad (46,7\%), aunque las edades comprendidas entre los 31 y 40 años (25\%) y los 41 y 50 años $(23,3 \%)$ son una franja también numerosa en los centros. Las edades inferiores a 30 años (5\%) son una minoría en este tipo de centros en la Ciudad Autónoma de Ceuta.

Los miembros participantes en el estudio llevan, en su mayoría, entre 11 y 20 años de docencia (50\%), suponiendo la mitad de la población. La titulación que más predomina entre los docentes estudiados es la diplomatura $(76,7 \%)$ y el motivo por el cual se incorporaron a las cooperativas de enseñanza es la vocación docente $(48,3 \%)$.

\section{Instrumento}

Para recoger los datos de este estudio se ha utilizado un cuestionario ad hoc, generado específicamente para esta investigación, tratando de dar respuesta a los objetivos planteados.

El cuestionario está formado por 57 cuestiones cerradas, distribuidas en: ámbito cooperativo, que tiene 13 preguntas; ámbito directivo, que consta de 7 preguntas; ámbito de formación complementaria, que está formado por 7 interrogantes; y ámbito liderazgo, con 25 preguntas. Al comienzo del instrumento se han incluido los datos sociodemográficos (género, edad, años de trabajo, grado de titulación académica y motivación laboral).

El instrumento ha sido validado, mediante validez de contenidos por cinco expertos de distintas instituciones andaluzas (Sevilla, Granada y Málaga). Su fiabilidad se ha comprobado mediante el coeficiente de consistencia interna de Alfa de Cronbach, cuya prueba piloto, con 25 sujetos, ofreció resultados superiores al 0,85 de media, considerando como fiable la prueba.

\section{Método de investigación y procedimiento}

El estudio aplicado es de tipo descriptivo y correlacional, basado en un análisis cuantitativo. Además, se han usado las variables "género" y "edad" con los ítems del ámbito de formación complementaria.Para poder recopilar los diversos datos, se presentan los aspectos que quieren desarrollar a los equipos directivos de las tres cooperativas de enseñanza y se solicitó su aprobación y colaboración, algo que se obtuvo al momento. La presentación se realizó en el mes de julio del año 2016.

Los responsables del centro indicaron que se realizasen las pruebas en el mes de septiembre del curso académico 2017-2018, momento en el que la carga de trabajo es menor y el profesorado se presenta más colaborativo a la recepción de este tipo de estudios. Además, este periodo permite un fácil acceso a la totalidad de los docentes, ya que todos se encuentran reunidos en el claustro de profesores.

En la celebración del claustro, se explicó a los docentes cómo debían rellenar el cuestionario, además de ofrecerle el enlace para su cumplimentación, ya que el instrumento fue elaborado mediante la herramienta de Google Formularios. 
La mayoría de los docentes utilizaron su propio teléfono móvil para resolver el cuestionario, pero una minoría solicitó un ordenador portátil, el cual se tenía preparado, ya que tenían dificultad para rellenarlo por el otro medio anteriormente citado.

El periodo temporal que dedicaron los docentes para su cumplimentación fue de unos 4050 minutos aproximadamente. En todo momento presentaron buena predisposición y no fue necesario resolver ninguna duda.

\section{Análisis de datos}

Una vez recopilados los datos, se tuvo que generar una hoja de cálculo Excel, para luego importarla en el programa IBM SPSS Statistics 20. Esto se realizó a falta de un mes de la celebración del claustro de profesores.

Con el programa estadístico se generaron tablas de contingencias para ver las relaciones entre las variables anteriormente indicadas, obteniendo las frecuencias y los porcentajes de los datos solicitados. Además, se identificó la relación entre las variables mediante la prueba de Chi Cuadrado. En los casos en los que hubo relación, se aplicó la prueba de Tau-b de Kendall y la Correlación de Spearman, para variables ordinales, identificando la fuerza de relación entre las variables.

\section{Resultados}

Los resultados obtenidos han sido analizados tanto desde la perspectiva descriptiva como desde la perspectiva correlacional.

\section{Análisis descriptivo}

No existen diferencias significativas desde el punto de vista del género a la hora de considerar importante la formación complementaria y el reciclaje en la figura del docente. Los resultados muestran que es valorado positivamente tanto por hombres $(73,3 \%)$ como por mujeres $(80 \%)$, tal y como se observa en la tabla 1.

Tabla 1. Relación entre el género y la importancia de la formación docente

\begin{tabular}{llllll}
\hline Género & \multicolumn{5}{c}{ ¿Considera importante la formación complementaria y el reciclaje de conocimientos } \\
en la figura docente?
\end{tabular}

Fuente: Elaboración propia.

Aunque consideran importante la formación complementaria, no disponen de tiempo para ampliar su formación, considerando tanto hombres $(46,7 \%)$ como mujeres $(46,7 \%)$ que poseen 
"poca" disponibilidad. A rasgos generales, son las mujeres de las cooperativas de enseñanza las que tienen un poco más de tiempo que los hombres (Tabla 2).

Tabla 2. Relación entre el género y tiempo para dedicar a la formación

\begin{tabular}{llllll}
\hline Género & \multicolumn{5}{c}{ ¿Dispone de tiempo para ampliar su formación (máster, postgrado, doctorado) mientras } \\
\cline { 3 - 6 } & & Nada & Poco & Solo en parte & Bastante \\
\multirow{2}{*}{ Hombre } & $\mathrm{N}$ & 3 & 7 & 3 & 2 \\
& $\%$ & $20 \%$ & $46,7 \%$ & $20 \%$ & $13,3 \%$ \\
\multirow{3}{*}{ Mujer } & $\mathrm{N}$ & 7 & 21 & 17 & 0 \\
& $\%$ & $15,6 \%$ & $46,7 \%$ & $37,8 \%$ & $0 \%$ \\
\hline
\end{tabular}

Fuente: Elaboración propia.

En la tabla 3, se observa que existen diferencias desde la perspectiva del género de los cooperativistas a la hora de considerar que la formación de los socios que acceden a las mismas es escasa desde un punto de vista empresarial. Los hombres señalaron "bastante" y "solo en parte" en un $40 \%$ de los casos, respectivamente. Por otro lado, las mujeres valoraron "bastante" $(26,7 \%)$ y "solo en parte" (33,3\%).

Tabla 3. Relación entre el género y la formación de nuevos cooperativistas

\begin{tabular}{|c|c|c|c|c|c|}
\hline \multirow[t]{2}{*}{ Género } & & \multicolumn{4}{|c|}{$\begin{array}{c}\text { ¿Cree que la formación de los socios que acceden a cooperativas de enseñanza es } \\
\text { escasa en aspectos cooperativos y términos empresariales? }\end{array}$} \\
\hline & & Nada & Poco & Solo en parte & Bastante \\
\hline \multirow[t]{2}{*}{ Hombre } & $\mathrm{N}$ & 1 & 2 & 6 & 6 \\
\hline & $\%$ & $6,7 \%$ & $13,3 \%$ & $40 \%$ & $40 \%$ \\
\hline \multirow[t]{2}{*}{ Mujer } & $\mathrm{N}$ & 5 & 13 & 15 & 12 \\
\hline & $\%$ & $11,1 \%$ & $28,9 \%$ & $33,3 \%$ & $26,7 \%$ \\
\hline
\end{tabular}

Fuente: Elaboración propia.

No existen diferencias significativas, en opinión de los cooperativistas, a la hora de considerar si el equipo directivo se preocupa de la formación relacionada con aspectos empresariales de sus miembros, siendo valorado como "solo en parte" tanto por hombres $(46,7$ $\%$ ) como por mujeres $(44,4 \%)$ como se refleja en la tabla 4.

Tabla 4. Relación entre el género y la preocupación del equipo directivo por la formación

\begin{tabular}{|c|c|}
\hline Género & $\begin{array}{l}\text { ¿Considera que el equipo directivo-administrativo se preocupa lo suficiente de la } \\
\text { formación relacionada con los aspectos empresariales y cooperativos de cara a la } \\
\text { preparación de sus socios? }\end{array}$ \\
\hline
\end{tabular}

\begin{tabular}{|c|c|c|c|c|c|}
\hline & & Nada & Poco & Solo en parte & Bastante \\
\hline \multirow{2}{*}{ Hombre } & $\mathrm{N}$ & 0 & 4 & 7 & 4 \\
\hline & $\%$ & $0 \%$ & $26,7 \%$ & $46,7 \%$ & $26,7 \%$ \\
\hline \multirow[t]{2}{*}{ Mujer } & $\mathrm{N}$ & 2 & 14 & 20 & 9 \\
\hline & $\%$ & $4,4 \%$ & $31,1 \%$ & $44,4 \%$ & $20 \%$ \\
\hline
\end{tabular}

Fuente: Elaboración propia. 
En la tabla 5 se observa que las mujeres $(44,4 \%)$ prefieren la modalidad presencial de cara a la de desarrollar una formación específica, al igual que los hombres $(33,3 \%)$, aunque en un porcentaje menor. Destaca la preferencia por parte de hombres $(26,7 \%)$ por realizar seminarios con respecto a las mujeres $(15,6 \%)$.

Tabla 5. Relación entre el género y la modalidad de cursos a realizar

\begin{tabular}{|c|c|c|c|c|c|}
\hline \multirow[t]{2}{*}{ Género } & & \multicolumn{4}{|c|}{ ¿Qué modalidad de cursos prefiere realizar? } \\
\hline & & Presencial & Semi-presencial & A distancia & Seminario \\
\hline \multirow[t]{2}{*}{ Hombre } & $\mathrm{N}$ & 5 & 3 & 3 & 4 \\
\hline & $\%$ & $33,3 \%$ & $20 \%$ & $20 \%$ & $26,7 \%$ \\
\hline \multirow[t]{2}{*}{ Mujer } & $\mathrm{N}$ & 20 & 10 & 8 & 7 \\
\hline & $\%$ & $44,4 \%$ & $22,2 \%$ & $17,8 \%$ & $15,6 \%$ \\
\hline
\end{tabular}

Fuente: Elaboración propia.

En aquellos casos en el que los miembros de la cooperativa de enseñanza se decantan por formarse, prefieren temas relacionados con el ámbito educativo, siendo semejante las necesidades tanto por parte de hombres $(66,7 \%)$ como de mujeres $(71,1 \%)$, tal como se observa en la tabla 6.

Tabla 6. Relación entre el género y el tipo de formación a realizar

\begin{tabular}{|c|c|c|c|c|c|}
\hline \multirow[t]{2}{*}{ Género } & & \multicolumn{4}{|c|}{$\begin{array}{l}\text { En el conjunto de la formación programada por el Centro para el curso escolar, ¿en } \\
\text { cuál de ellos se implica más? }\end{array}$} \\
\hline & & Ámbito educativo & Nuevas tecnologías & Idiomas & Ámbito administrativo \\
\hline \multirow[t]{2}{*}{ Hombre } & $\mathrm{N}$ & 10 & 3 & 2 & 0 \\
\hline & $\%$ & $66,7 \%$ & $20 \%$ & $13.3 \%$ & $0 \%$ \\
\hline \multirow[t]{2}{*}{ Mujer } & $\mathrm{N}$ & 32 & 7 & 4 & 2 \\
\hline & $\%$ & $71,1 \%$ & $15,6 \%$ & $8,9 \%$ & $4,4 \%$ \\
\hline
\end{tabular}

Fuente: Elaboración propia.

Existen diferencias en el número de cursos realizados a nivel de género por parte de los cooperativistas. Los hombres, o no realizan cursos $(40 \%)$ o realizan entre $1-2(53,3 \%)$ por año académico. En cambio, las mujeres hacen entre 1-2 (71,1\%) por curso escolar, en un porcentaje mayor que los hombres (Tabla 7).

Tabla 7. Relación entre el género y el número de cursos a realizar

\begin{tabular}{|c|c|c|c|c|c|}
\hline \multirow[t]{2}{*}{ Género } & & \multicolumn{4}{|c|}{ ¿Cuántos cursos de formación suele realizar cada año? } \\
\hline & & Ninguno & $1-2$ & $3-4$ & Más de 5 \\
\hline \multirow[t]{2}{*}{ Hombre } & $\mathrm{N}$ & 6 & 8 & 1 & 0 \\
\hline & $\%$ & $40 \%$ & $53,3 \%$ & $6,7 \%$ & $0 \%$ \\
\hline \multirow[t]{2}{*}{ Mujer } & $\mathrm{N}$ & 8 & 32 & 4 & 1 \\
\hline & $\%$ & $17,8 \%$ & $71,1 \%$ & $8,9 \%$ & $1,7 \%$ \\
\hline
\end{tabular}

Fuente: Elaboración propia. 
Con respecto a la edad de los cooperativistas de enseñanza a la hora de considerar importante la formación complementaria y el reciclaje en la figura docente, se observa que no existen diferencias significativas en las valoraciones, siendo consideradas como "bastante", estando mejor considerado por las edades comprendidas entre 20-30 años (100\%) y 41-50 años $(85,7 \%)$, como se muestra en la tabla 8 .

Tabla 8. Relación entre la edad y la importancia de la formación docente

\begin{tabular}{|c|c|c|c|c|c|}
\hline \multirow[t]{2}{*}{ Edad } & & \multicolumn{4}{|c|}{$\begin{array}{c}\text { ¿Considera importante la formación complementaria y el reciclaje de conocimientos } \\
\text { en la figura docente? }\end{array}$} \\
\hline & & Nada & Poco & Solo en parte & Bastante \\
\hline \multirow[t]{2}{*}{$20-30$} & $\mathrm{~N}$ & 0 & 0 & 0 & 3 \\
\hline & $\%$ & $0 \%$ & $0 \%$ & $0 \%$ & $100 \%$ \\
\hline \multirow[t]{2}{*}{$31-40$} & $\mathrm{~N}$ & 0 & 1 & 4 & 10 \\
\hline & $\%$ & $0 \%$ & $6,7 \%$ & $26,7 \%$ & $66,7 \%$ \\
\hline \multirow[t]{2}{*}{$41-50$} & $\mathrm{~N}$ & 0 & 2 & 12 & 14 \\
\hline & $\%$ & $0 \%$ & $0 \%$ & $14,3 \%$ & $85,7 \%$ \\
\hline \multirow{2}{*}{$\begin{array}{l}\text { Más de } \\
\text { 50 }\end{array}$} & $\mathrm{N}$ & 0 & 2 & 4 & 22 \\
\hline & $\%$ & $0 \%$ & $7,1 \%$ & $14,3 \%$ & $78,6 \%$ \\
\hline
\end{tabular}

Fuente: Elaboración propia.

Como se observa en la tabla 9, en cuanto a si disponen de tiempo para ampliar su formación mientras ejercen la docencia, las valoraciones son semejantes entre las distintas edades, siendo "poca", aunque con porcentajes más altos en las franjas de 20-30 años (66,7 \%) y 41-50 años $(64,3 \%)$.

Tabla 9. Relación entre la edad y tiempo para dedicar a la formación

\begin{tabular}{|c|c|c|c|c|c|}
\hline \multirow[t]{2}{*}{ Edad } & & \multicolumn{4}{|c|}{$\begin{array}{c}\text { ¿Dispone de tiempo para ampliar su formación (máster, postgrado, doctorado) } \\
\text { mientras ejerce de docente? }\end{array}$} \\
\hline & & Nada & Poco & Solo en parte & Bastante \\
\hline \multirow[t]{2}{*}{$20-30$} & $\mathrm{~N}$ & 0 & 2 & 1 & 0 \\
\hline & $\%$ & $0 \%$ & $66,7 \%$ & $33,3 \%$ & $0 \%$ \\
\hline \multirow[t]{2}{*}{$31-40$} & $\mathrm{~N}$ & 5 & 7 & 3 & 0 \\
\hline & $\%$ & $33,3 \%$ & $46,7 \%$ & $20 \%$ & $0 \%$ \\
\hline \multirow[t]{2}{*}{$41-50$} & $\mathrm{~N}$ & 2 & 9 & 3 & 0 \\
\hline & $\%$ & $14,3 \%$ & $64,3 \%$ & $21,4 \%$ & $0 \%$ \\
\hline Más de & $\mathrm{N}$ & 3 & 10 & 13 & 2 \\
\hline $\mathbf{5 0}$ & $\%$ & $16,7 \%$ & $46,7 \%$ & $33,3 \%$ & $3,3 \%$ \\
\hline
\end{tabular}

Fuente: Elaboración propia.

En la tabla 10, las valoraciones son más dispersas en relación a la creencia de que la formación de los socios cooperativistas es escasa en aspectos cooperativos y términos empresariales. Son los docentes con edades comprendidas entre 20-30 años (33,3 \%) y 31-40 años $(33,3 \%)$ los que lo valoran como "bastante", mientras que las edades comprendidas entre los 41-50 años (42,9\%) y los que tienen más de 50 años $(35,7 \%)$ consideran que "solo en parte". 
Tabla 10. Relación entre la edad y la formación de nuevos cooperativistas

\begin{tabular}{llllll} 
Edad & \multicolumn{5}{c}{$\begin{array}{c}\text { iCree que la formación de los socios que acceden, a cooperativas de enseñanza, es } \\
\text { escasa en aspectos cooperativos y términos empresariales? }\end{array}$} \\
\cline { 3 - 6 } & & \multicolumn{7}{c}{ Pada } & Poco & Solo en parte & Bastante \\
$\mathbf{2 0}-\mathbf{3 0}$ & $\mathrm{N}$ & 0 & 1 & 1 & 1 \\
& $\%$ & $0 \%$ & $33,3 \%$ & $33,3 \%$ & $33,3 \%$ \\
$\mathbf{3 1}-\mathbf{4 0}$ & $\mathrm{N}$ & 2 & 4 & 4 & 5 \\
& $\%$ & $13,3 \%$ & $26,7 \%$ & $26,7 \%$ & $33,3 \%$ \\
$\mathbf{4 1}-\mathbf{5 0}$ & $\mathrm{N}$ & 1 & 3 & 6 & 4 \\
& $\%$ & $7,1 \%$ & $21,4 \%$ & $42,9 \%$ & $28,6 \%$ \\
Más de & $\mathrm{N}$ & 6 & 5 & 21 & 18 \\
$\mathbf{5 0}$ & $\%$ & $10,7 \%$ & $25 \%$ & $35,7 \%$ & $28,6 \%$
\end{tabular}

Fuente: Elaboración propia.

En relación al hecho de considerar si el equipo directivo se preocupa lo suficiente en la formación relacionada con aspectos empresariales y cooperativos de cara a la preparación de sus socios existen discrepancias en función a la franja de edad de los cooperativistas. Para los que tienen entre 20 y 30 años de edad, sus valoraciones se reparten entre "poco", "solo en parte" y "bastante" con un porcentaje del 33,3\%. Los que se sitúan en edades comprendidas entre los 31 y 40 años $(47,6 \%)$ y los que tienen más de 50 años $(53,6 \%)$, valoran que "solo en parte". Aquellos que tienen entre 41 y 50 años edad creen que "poco" (50\%), tal y como se observa en la tabla 11.

Tabla 11. Relación entre la edad y la preocupación del equipo directivo por la formación

\begin{tabular}{|c|c|c|c|c|c|}
\hline \multirow[t]{2}{*}{ Edad } & & \multicolumn{4}{|c|}{$\begin{array}{l}\text { ¿Considera que el equipo directivo-administrativo se preocupa lo suficiente de la } \\
\text { formación relacionada con los aspectos empresariales y cooperativos de cara a la } \\
\text { preparación de sus socios? }\end{array}$} \\
\hline & & Nada & Poco & Solo en parte & Bastante \\
\hline $20-$ & $\mathrm{N}$ & 0 & 1 & 1 & 1 \\
\hline 30 & $\%$ & $0 \%$ & $33,3 \%$ & $33,3 \%$ & $33,3 \%$ \\
\hline $31-$ & $\mathrm{N}$ & 1 & 2 & 7 & 5 \\
\hline 40 & $\%$ & $6,7 \%$ & $13,3 \%$ & $46,7 \%$ & $33,3 \%$ \\
\hline $41-$ & $\mathrm{N}$ & 0 & 7 & 4 & 3 \\
\hline 50 & $\%$ & $0 \%$ & $50 \%$ & $28,6 \%$ & $21,4 \%$ \\
\hline Más & $\mathrm{N}$ & 1 & 8 & 15 & 4 \\
\hline de 50 & $\%$ & $3,6 \%$ & $28,6 \%$ & $53,6 \%$ & $14,3 \%$ \\
\hline
\end{tabular}

Fuente: Elaboración propia.

Con respecto al tipo de formación que prefieren realizar, tal y como se refleja en la tabla 12, las edades comprendidas entre los 20 y 30 años edad son más flexibles, considerando la modalidad presencial, a distancia y seminario por partes iguales $(33,3 \%)$. Los que tienen entre 31 y 40 años de edad prefieren la modalidad semipresencial $(33,3 \%)$ y a distancia $(40 \%)$. Las 
edades entre 41 y 50 años se decantan por la formación presencial $(35,7 \%)$ y semipresencial $(42,9 \%)$. Aquellos con más de 50 años prefieren claramente la formación presencial $(57,1 \%)$.

Tabla 12. Relación entre la edad y la modalidad de cursos a realizar

\begin{tabular}{|c|c|c|c|c|c|}
\hline \multirow[t]{2}{*}{ Edad } & & \multicolumn{4}{|c|}{ ¿Qué modalidad de cursos prefiere realizar? } \\
\hline & & Presencial & Semipresencial & A distancia & Seminario \\
\hline \multirow[t]{2}{*}{$\mathbf{2 0}-\mathbf{3 0}$} & $\mathrm{N}$ & 1 & 0 & 1 & 1 \\
\hline & $\%$ & $33,3 \%$ & $0 \%$ & $33,3 \%$ & $33,3 \%$ \\
\hline \multirow[t]{2}{*}{$31-40$} & $\mathrm{~N}$ & 3 & 5 & 6 & 1 \\
\hline & $\%$ & $20 \%$ & $33,3 \%$ & $40 \%$ & $6,7 \%$ \\
\hline \multirow[t]{2}{*}{$41-50$} & $\mathrm{~N}$ & 5 & 6 & 0 & 3 \\
\hline & $\%$ & $35,7 \%$ & $42,9 \%$ & $0 \%$ & $21,4 \%$ \\
\hline \multirow[t]{2}{*}{ Más de 50} & $\mathrm{~N}$ & 16 & 2 & 4 & 6 \\
\hline & $\%$ & $57,1 \%$ & $7,1 \%$ & $14,3 \%$ & $21,4 \%$ \\
\hline
\end{tabular}

Fuente: Elaboración propia.

Los cooperativistas de enseñanza se decantan por una formación más centrada en el ámbito educativo, principalmente en las edades comprendidas entre los 31-40 años (73,3 \%), 4150 años $(92,9 \%)$ y con más de 50 años $(60,7 \%)$, aunque los más jóvenes valoran también la formación en idiomas y en el ámbito administrativo, como se visualiza en la tabla 13.

Tabla 13. Relación entre la edad y el tipo de formación a realizar

\begin{tabular}{llllll}
\hline Edad & \multicolumn{5}{c}{ En el conjunto de la formación programada por el Centro para el curso escolar, ¿en } \\
& & & \multicolumn{4}{c}{\begin{tabular}{c} 
cuál de ellos se implica más? \\
\cline { 3 - 6 }
\end{tabular}} & & Ámbito educativo & Nuevas tecnologías & Idiomas & Ámbito administrativo \\
$\mathbf{2 0 - 3 0}$ & $\mathrm{N}$ & 1 & 0 & 1 & 1 \\
& $\%$ & $33,3 \%$ & $0 \%$ & $33,3 \%$ & $33,3 \%$ \\
$\mathbf{3 1 - 4 0}$ & $\mathrm{N}$ & 11 & 2 & 2 & 0 \\
& $\%$ & $73,3 \%$ & $13,3 \%$ & $13,3 \%$ & $0 \%$ \\
$\mathbf{4 1 - 5 0}$ & $\mathrm{N}$ & 13 & 1 & 0 & 0 \\
& $\%$ & $92,9 \%$ & $7,1 \%$ & $0 \%$ & $0 \%$ \\
Más de & $\mathrm{N}$ & 17 & 7 & 3 & 1 \\
$\mathbf{5 0}$ & $\%$ & $60,7 \%$ & $25 \%$ & $10,7 \%$ & $3,6 \%$ \\
\hline
\end{tabular}

Fuente: Elaboración propia.

Finalmente, en relación al número de cursos de formación realizados durante el curso escolar, aquellos que tienen entre 20 y $30(66,7 \%)$, entre 31 y $40(73,3 \%)$, entre 41 y $50(71,4$ $\%)$, y más de 50 años $(60,7 \%)$ realizan entre 1-2 cursos de formación al año, como se muestra en la tabla 14.

Tabla 14. Relación entre la edad y el número de cursos a realizar

\begin{tabular}{llllll}
\hline \multirow{2}{*}{ Edad } & \multicolumn{4}{c}{ ¿Cuántos cursos de formación suele realizar cada año? } \\
\cline { 3 - 6 } & & Ninguno & $1-2$ & $3-4$ & Más de 5 \\
$\mathbf{2 0}-\mathbf{3 0}$ & $\mathrm{N}$ & 0 & 2 & 1 & 0 \\
& $\%$ & $0 \%$ & $66,7 \%$ & $33,3 \%$ & $0 \%$ \\
$\mathbf{3 1 - 4 0}$ & $\mathrm{N}$ & 4 & 11 & 0 & 0 \\
\hline
\end{tabular}




\begin{tabular}{llllll}
\hline \multirow{4}{41-50}{} & $\%$ & $26,7 \%$ & $73,3 \%$ & $0 \%$ & $0 \%$ \\
& $\mathrm{~N}$ & 3 & 10 & 1 & 0 \\
\multirow{4}{*}{ Más de 50 } & $\%$ & $21,4 \%$ & $71,4 \%$ & $7,1 \%$ & $0 \%$ \\
& $\mathrm{~N}$ & 7 & 17 & 3 & 1 \\
& $\%$ & $25 \%$ & $60,7 \%$ & $10,7 \%$ & $3,6 \%$ \\
\hline
\end{tabular}

Fuente: Elaboración propia.

\section{Análisis correlacional}

Para el análisis correlacional se han establecido una serie de hipótesis en relación a las distintas variables analizadas, con respecto a los ítems "género" y "edad", respectivamente.

H1: "Hay diferencias entre hombres y mujeres a la hora de considerar importante la formación complementaria y el reciclaje de conocimientos en la figura docente".

Resultados: Se acepta la hipótesis de independencia entre las dos variables. Se obtiene un p-valor en la prueba de Chi-Cuadrado de Pearson de 0,226.

Interpretación: El hecho de ser hombre o mujer no influye a la hora de ser considerar importante la formación complementaria y el reciclaje de conocimientos en la figura docente.

H2: "Hay diferencias entre hombres y mujeres a la hora de determinar si disponen de tiempo para ampliar su formación mientras ejercen la docencia”.


p-valor en la prueba de Chi-Cuadrado de Pearson de 0,066.

Interpretación: El hecho de ser hombre o mujer no influye a la hora de determinar si disponen de tiempo para ampliar su formación mientras ejercen la docencia.

H3: "Hay diferencias entre hombres y mujeres a la hora de determinar si la formación de los socios que acceden a las cooperativas de enseñanza es escasa en aspectos cooperativos y términos empresariales".

Resultados: Se acepta la hipótesis de independencia entre las dos variables. Se obtiene un p-valor en la prueba de Chi-Cuadrado de Pearson de 0,548.

Interpretación: El hecho de ser hombre o mujer no influye a la hora de determinar si la formación de los socios que acceden a las cooperativas de enseñanza es escasa en aspectos cooperativos y términos empresariales.

H4: "Hay diferencias entre hombres y mujeres a la hora de determinar si el equipo directivo se preocupa lo suficiente de la formación relacionada con aspectos empresariales y cooperativas de cara a la preparación de sus socios".

Resultados: Se acepta la hipótesis de independencia entre las dos variables. Se obtiene un p-valor en la prueba de Chi-Cuadrado de Pearson de 0,805.

Interpretación: El hecho de ser hombre o mujer no influye a la hora de determinar si el equipo directivo se preocupa lo suficiente de la formación relacionada con aspectos empresariales y cooperativas de cara a la preparación de sus socios. 
H5: "Hay diferencias entre hombres y mujeres a la hora de elegir la modalidad de cursos de formación que prefiere realizar".

Resultados: Se acepta la hipótesis de independencia entre las dos variables. Se obtiene un p-valor en la prueba de Chi-Cuadrado de Pearson de 0,766.

Interpretación: El hecho de ser hombre o mujer no influye a la hora de elegir la modalidad de cursos de formación que prefiere realizar.

H6: "Hay diferencias entre hombres y mujeres a la hora de elegir el tipo de formación que prefiere realizar".

Resultados: Se acepta la hipótesis de independencia entre las dos variables. Se obtiene un p-valor en la prueba de Chi-Cuadrado de Pearson de 0,788.

Interpretación: El hecho de ser hombre o mujer no influye a la hora de elegir el tipo de formación que prefiere realizar.

H7: "Hay diferencias entre hombres y mujeres a la hora de realizar un número determinados de cursos de formación cada año".

Resultados: Se acepta la hipótesis de independencia entre las dos variables. Se obtiene un p-valor en la prueba de Chi-Cuadrado de Pearson de 0,346.

Interpretación: El hecho de ser hombre o mujer no influye a la hora de realizar un número determinados de cursos de formación cada año.

Tabla 15. Fuerza relación entre género y variables. Chi Cuadrado

\begin{tabular}{|c|c|}
\hline Variables & $\begin{array}{c}\mathrm{P}- \\
\text { valor }\end{array}$ \\
\hline $\begin{array}{l}\text { ¿Considera importante la formación complementaria y el reciclaje de conocimientos en la figura } \\
\text { docente? }\end{array}$ & 0,226 \\
\hline $\begin{array}{l}\text { ¿Dispone de tiempo para ampliar su formación (máster, postgrado, doctorado) mientras ejerce de } \\
\text { docente? }\end{array}$ & 0,066 \\
\hline $\begin{array}{l}\text { ¿Cree que la formación de los socios que acceden, a cooperativas de enseñanza, es escasa en } \\
\text { aspectos cooperativos y términos empresariales? }\end{array}$ & 0,548 \\
\hline ¿Considera que el equipo directivo-administrativo se preocupa lo suficiente de la formación & 0,805 \\
\hline relacionada con los aspectos empresariales y cooperativos de cara a la preparación de sus socios? & \\
\hline $\begin{array}{l}\text { En el conjunto de la formación programada por el Centro para el curso escolar, ¿en cuál de ellos se } \\
\text { implica más? }\end{array}$ & $\begin{array}{l}0,766 \\
0,788\end{array}$ \\
\hline ¿Cuántos cursos de formación suele realizar cada año? & 0,346 \\
\hline
\end{tabular}

Fuente: Elaboración propia.

H8: "Hay diferencias en la edad de los cooperativistas a la hora de considerar importante la formación complementaria y el reciclaje de conocimientos en la figura docente".

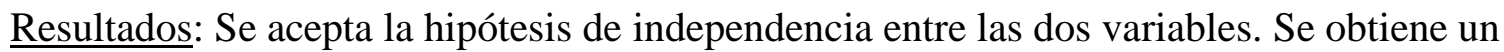
p-valor en la prueba de Chi-Cuadrado de Pearson de 0,774.

Interpretación: El hecho de tener una determinada edad no influye a la hora de ser considerar importante la formación complementaria y el reciclaje de conocimientos en la figura docente. 
H9: "Hay diferencias en la edad de los cooperativistas a la hora de determinar si disponen de tiempo para ampliar su formación mientras ejercen la docencia”.

Resultados: Se acepta la hipótesis de independencia entre las dos variables. Se obtiene un p-valor en la prueba de Chi-Cuadrado de Pearson de 0,299.

Interpretación: El hecho de tener una determina edad no influye a la hora de determinar si disponen de tiempo para ampliar su formación mientras ejercen la docencia.

H10: "Hay diferencias en la edad de los cooperativistas a la hora de determinar si la formación de los socios que acceden a las cooperativas de enseñanza es escasa en aspectos cooperativos y términos empresariales".

Resultados: Se acepta la hipótesis de independencia entre las dos variables. Se obtiene un p-valor en la prueba de Chi-Cuadrado de Pearson de 0,998.

Interpretación: El hecho de tener una determinada edad no influye a la hora de determinar si la formación de los socios que acceden a las cooperativas de enseñanza es escasa en aspectos cooperativos y términos empresariales.

H11: "Hay diferencias en la edad de los cooperativistas a la hora de determinar si el equipo directivo se preocupa lo suficiente de la formación relacionada con aspectos empresariales y cooperativas de cara a la preparación de sus socios”.


p-valor en la prueba de Chi-Cuadrado de Pearson de 0,576.

Interpretación: El hecho de tener una determinada edad no influye a la hora de determinar si el equipo directivo se preocupa lo suficiente de la formación relacionada con aspectos empresariales y cooperativas de cara a la preparación de sus socios.

H12: "Hay diferencias en la edad de los cooperativistas a la hora de elegir la modalidad de cursos de formación que prefiere realizar".

Resultados: Se rechaza la hipótesis de independencia entre las dos variables. Se obtiene un p-valor en la prueba de Chi-Cuadrado de Pearson de 0,022.

Interpretación: El hecho de tener una determinada edad influye a la hora de elegir la modalidad de cursos de formación que prefiere realizar, en este caso, las personas con edades superiores a los 31 años de edad no necesariamente se decantarán por cursos de formación relacionados con el ámbito educativo.

Fuerza de asociación: Los resultados obtenidos muestran una fuerza de asociación débil y negativa, dando a interpretación lo indicado anteriormente.

Tabla 16. Relación entre modalidad de cursos y la edad

\begin{tabular}{ll}
\hline Tau-B de Kendall & $\mathbf{- 0 , 1 7 2}$ \\
\hline Correlación de Spearman & $-0,185$ \\
\hline
\end{tabular}

Fuente: Elaboración propia.

H13: "Hay diferencias en la edad de los cooperativistas a la hora de elegir el tipo de formación que prefiere realizar". 
Resultados: Se acepta la hipótesis de independencia entre las dos variables. Se obtiene un p-valor en la prueba de Chi-Cuadrado de Pearson de 0,052.

Interpretación: El hecho de tener una determinada edad no influye a la hora de elegir el tipo de formación que prefiere realizar.

H14: "Hay diferencias en la edad de los cooperativistas a la hora de realizar un número determinados de cursos de formación cada año".

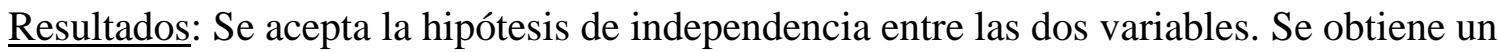
p-valor en la prueba de Chi-Cuadrado de Pearson de 0,742.

Interpretación: El hecho de tener una determinada edad no influye a la hora de realizar un número determinados de cursos de formación cada año.

Tabla 17. Fuerza relación entre edad y variables. Chi Cuadrado

\begin{tabular}{lc}
\hline Variables & $\begin{array}{c}\text { P - } \\
\text { valor }\end{array}$ \\
\hline ¿Considera importante la formación complementaria y el reciclaje de conocimientos en la figura & 0,774 \\
docente? & 0,299 \\
¿Dispone de tiempo para ampliar su formación (máster, postgrado, doctorado) mientras ejerce de & \\
docente? & 0,998 \\
¿Cree que la formación de los socios que acceden, a cooperativas de enseñanza, es escasa en & \\
aspectos cooperativos y términos empresariales? & 0,576 \\
¿Considera que el equipo directivo-administrativo se preocupa lo suficiente de la formación & \\
relacionada con los aspectos empresariales y cooperativos de cara a la preparación de sus socios? & \\
¿Qué modalidad de cursos prefiere realizar? & 0,022 \\
En el conjunto de la formación programada por el Centro para el curso escolar, ¿en cuál de ellos se & 0,052 \\
implica más? & 0,742 \\
¿Cuántos cursos de formación suele realizar cada año?
\end{tabular}

Fuente: Elaboración propia.

\section{Discusión}

Una vez realizada la interpretación de cada una de las tablas de datos originadas, se procede a extraer la información apoyada en autores que han realizado estudios sobre la temática abordada en esta investigación. En un primer orden, los hombres y mujeres de las cooperativas de enseñanza valoran positivamente la formación complementaria, como afirmó Marcelo (2002), los docentes necesitan actualizar sus conocimientos para ser mejores profesionales, pero los sujetos analizados de las distintas cooperativas de enseñanza se encuentran ante la dificultad de no disponer del tiempo suficiente para poder formarse. Este factor temporal hace que no se pueda desarrollar un programa formativo pleno, como determinó Andreucci (2012), que sirva para romper las estructuras de la monotonía y el estancamiento docente.

Los miembros de las cooperativas de enseñanza, tanto hombres como mujeres, establecen que los cooperativistas no tienen una formación adecuada en el ámbito empresarial, algo que, desde la perspectiva de dichos miembros, también percibe el equipo directivo. En esta línea, tomando de referencia los estudios realizados por Fuentes (2004), toda cooperativa requiere que sus profesionales estén debidamente formados tanto en el plano educativo como empresarial, para llevar a cabo una gestión eficaz de la entidad y, por consiguiente, una mejora de la calidad, siguiendo lo marcado por Mayorga, Santos y Madrid (2014). 
A la hora de formarse, las mujeres se decantan por la formación presencial, al igual que los hombres, aunque estos últimos también se declinan por los seminarios, prefiriendo formarse ambos géneros en aspectos relacionados con el ámbito educativo. Resulta pertinente puntualizar que las mujeres tienden a realizar más cursos de formación que los hombres.

En relación a la edad de los cooperativistas, éstos valoran positivamente la necesidad de la formación del profesorado, siendo los docentes con edades entre los 20-30 años y los 31-40 años de edad los que menos tiempo tienen para poder realizar cursos de formación. A pesar del tiempo disponible, la motivación de las personas es un aspecto fundamental para poder realizar este tipo de formación, tal y como recogen Muñoz-Repiso y Murillo (2010).

Existen diferencias entre aquellos que tienen menos de 40 años con respecto a los que tienen más de 40 años en relación a considerar la formación de los miembros de la cooperativa de enseñanza en aspectos empresariales. Los primeros consideran que hay una formación muy escasa, mientras que los segundos consideran que hay falta de formación, aunque no tan acuciada.

Para los cooperativistas con edades comprendidas entre los 31-50 años, su equipo directivo se preocupa porque los miembros del claustro dispongan de formación en el ámbito empresarial. En cambio, los que tienen entre 20-30 y los de más de 50 años piensan que su equipo directivo se preocupa poco en ese aspecto. Aquí es donde entra en juego el liderazgo pedagógico analizado por González, Gento y Orden (2016), en el caso que los órganos unipersonales deben promover este ámbito de la formación permanente tan importante en las cooperativas de enseñanza.

La modalidad de formación que prefieren los cooperativistas de enseñanza es muy variada, siendo los mayores de 50 años los que más se postulan al respecto, prefiriendo la modalidad presencial.

Todos los cooperativistas de enseñanza de cualquier edad prefieren la formación en el ámbito educativo, aunque los más jóvenes también se decantan por los idiomas y el ámbito administrativo, realizando una media de uno o dos cursos por año académico. Estas temáticas coinciden con los estudios de Fuentes (2004), Lobato y Madinabeitia (2011); Pontes, Serrano y Poyato (2013); Alfaro, Fernández y Alvarado (2014); Gisbert y Lázaro (2015).

Concretamente en este estudio, no hay relación entre las variables género y las variables analizadas, ocurriendo lo mismo con la edad, aunque hay una excepción en el hecho de la edad y la modalidad de cursos de formación que prefieren realizar, en la que se observa que el hecho de tener más de 31 años de edad no necesariamente hará que los profesores se decanten por cursos de formación relacionados con el ámbito educativo.

\section{Conclusión}

Se concluye que los docentes que forman parte de las cooperativas de enseñanza le dan importancia a la necesidad de una formación continua, aunque destacan que no disponen de tiempo para realizar cursos de perfeccionamiento, sobre todo las personas menores de 40 años. 
Aunque consideran que no tienen una formación adecuada para el ámbito empresarial por formar parte de una cooperativa de enseñanza, no es éste el tipo de formación que reclaman, sino al contrario, buscan formarse en ámbitos pedagógicos, más relacionados con su tarea diaria.

Los hombres y las mujeres se decantan por la formación presencial, sobre todo los mayores de cincuenta años, aunque a la hora de cuantificar la cantidad de cursos de formación, las mujeres realizan más que los hombres.

No existe una correlación entre las variables analizadas en el estudio y los ítems edad y género, considerando que dichos elementos no influyen en la formación de los miembros de las cooperativas de enseñanza.

Como futura línea de investigación, se propone ampliar el estudio a centros educativos públicos y privados, para establecer una comparativa al respecto, tratando de observar si los resultados obtenidos son específicos de las cooperativas de enseñanza o es algo extrapolable a todos los centros educativos, independientemente de su naturaleza como centro escolar.

\section{Bibliografía}

Alfaro, A. P., Fernández, M. S., y Alvarado, R. I. (2014). El uso de las TIC en la formación permanente del profesorado para la mejora de su práctica docente.Etic@ net, 1 (14), 70-95.

Andreucci, P. M. (2012). El enfoque clínico en la formación continua de profesores: la teorización del «ojo pedagógico» como destreza compleja. Profesorado. Revista de Currículum y Formación de Profesorado, 16 (1), 257-275.

Casanova, M. A. (2012). El diseño curricular como factor de calidad educativa. REICE. Revista Iberoamericana sobre Calidad, Eficacia y Cambio en Educación, 10 (4), 6-20.

Escudero, J. M., Cutanda, M. T., y Trillo, J. F. (2017). Aprendizaje docente y desarrollo profesional del profesorado. Profesorado, Revista de Currículum y Formación del Profesorado, 21 (3), 83-102.

Fuentes, A. (2004). Las cooperativas de enseñanza:(un estudio de las cooperativas de trabajo asociado). Ceuta: Universidad Nacional de Educación a Distancia, Centro Asociado de Ceuta.

García, M. (2015). La educación actual: retos para el profesorado. Revista Ibero-Americana de Estudos em Educação, 10 (4), 1199-1211.

Gisbert, M., y Lázaro, J. L. (2015). La formación permanente en competencia digital docente y la mejora de la calidad del centro educativo desde la perspectiva de los docentes: un estudio de caso. Journal of New Approaches in Educational Research, 4 (2), 115-122.

González, G., y Barba, J. J. (2014). Formación permanente y desarrollo de la identidad reflexiva del profesorado desde las perspectivas grupal e individual. Profesorado. Revista de Currículum y Formación de Profesorado, 18 (1), 397-412.

González, R., Gento, S., y Orden, V. J. (2016). Importancia de la dimensión formativa de los líderes pedagógicos. Revista Ibero-americana de Educação, 70, 131-144. 
González, M. T., y Cutanda, M. T. (2017). Formación continuada del profesorado, mejora de la enseñanza y de los aprendizajes del alumnado. Revista Mexicana de Investigación Educativa, 22 (75), 1095 1116.

Lobato, C., y Madinabeitia, A. (2011). Perfiles motivacionales del profesorado ante la formación en metodologías activas en la universidad. Formación universitaria, 4(1), 37-48.

López, J. (2017). El fenómeno del liderazgo en los centros de enseñanza de naturaleza cooperativa. En Alcántara, J.M., Bermúdez, M., Blanco, F.J., y Heredia, J.M. (Eds.), Investigación e innovación en el ámbito universitario. Tendencias ante los retos actuales de la sociedad (pp. 37-46). Madrid, España: EOS.

López, J., y Fuentes, A. (2018). El liderazgo aplicado a los modelos diferenciados en educación: El caso de las cooperativas de enseñanza. En Gairín, J., y Mercader, C. (Eds.), Liderazgo y gestión del talento en las organizaciones (pp. 169-175). Madrid, España: Wolters Kluwer

Marcelo, C. (2002). Los profesores como trabajadores del conocimiento. Educar (30), 27-56.

Martínez, J. (2004). La formación del profesorado y el discurso de las competencias. Revista interuniversitaria de formación del profesorado, 18 (3), 127-143.

Mayorga, M. J., Santos, M. A, y Madrid, D. (2014). Formación y actualización de la función docente. Diálogos pedagógicos, 12 (24), 11-28.

Montes, M., y Torres, J. A. (2017). Las competencias socio-afectivas docentes y la formación para la práctica educativa del desarrollo personal y para la convivencia, en el marco de la educación inclusiva. Revista de Educación Inclusiva, 8 (3), 271-284.

Muñoz-Repiso, M., y Murillo, F. J. (2010). Un balance provisional sobre la calidad en educación: eficacia escolar y mejora de la escuela. REICE. Revista Iberoamericana sobre Calidad, Eficacia y Cambio en Educación, 8 (2), 177-186.

Nieto, J. M., y Alfageme, M. B. (2017). Enfoques, metodologías y actividades de formación docente. Profesorado, Revista de Currículum y Formación del Profesorado, 21 (3), 63-81.

Noguera, A. E. (2017). Consideraciones teóricas implícitas en la formación docente. Dialéctica, (1), 160190

Pontes, A., Serrano, R., y Poyato, F. J. (2013). Concepciones y motivaciones sobre el desarrollo profesional docente en la formación inicial del profesorado de educación secundaria. Revista Eureka sobre enseñanza y divulgación de las ciencias, (10), 533-551.

San Martín, A., Peirats, J., y Waliño, M. J. (2017). La formación docente y los sueños sobre el aula 3.0. Revista Internacional de Formação de Professores, 2 (1), 75-94. 